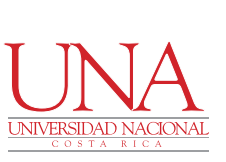

\title{
Comunidad de algas del manglar de playa Estrella, isla Colón, Bocas del Toro, Panamá
}

\section{Algae community of the mangrove at Playa Estrella, Colón Island, Bocas del Toro, Panama}

\author{
Rosemary Rios $^{1}$, Olga L. Tejada ${ }^{2 *}$, Diana Morales ${ }^{1}$ Elvia Miranda $^{1}$ y Angélica Cerrud ${ }^{1}$
}

\begin{abstract}
RESUMEN
Desarrollamos un estudio exploratorio descriptivo, de las especies de macroalgas que crecen asociadas a las raíces de $R$. mangle en playa Estrella, isla Colón, Bocas del Toro, Panamá, en agosto de 2012. El objetivo principal del estudio fue recolectar y describir las especies de algas encontradas y conocer la distribución vertical que forman en sus raíces. Para conocer la zonación vertical, en cada raíz se crearon tres zonas: 1) zona baja, desde la base de la raíz hasta $0.50 \mathrm{~m}$ de altura, 2) zona media de $0.50 \mathrm{~m}$ a $1.10 \mathrm{~m}$ y 3) zona alta, de 1.10 a $1.30 \mathrm{~m}$., de acuerdo con Peña-Salamanca (2008). Se identificaron once especies del phylum Rhodophyta, de las cuales B. montagnei, B. moritziana, B. tenella, Polysiphonia howei, P. scopulorum, A. spicifera y C. caespitosa fueron las más sobresalientes. Del phylum Chlorophyta se recolectaron e identificaron tres especies: C. linum, B. verticillata y Chlorodesmis sp. Respecto a la distribución vertical establecida por las algas en las raíces, la mayoría de especies se distribuyeron en las zonas baja y media y solamente el alga $B$. montagnei en la parte alta. Con el estudio se espera contribuir al conocimiento de la ficoflora de los manglares del Caribe de Panamá.
\end{abstract}

Palabras clave: Bostrichietum, raíces, distribución vertical, tolerancia a la salinidad, mar Caribe

\begin{abstract}
A descriptive exploratory study was conducted on macroalgae species associated to roots of $R$. mangle in Playa Estrella, Colón Island, Bocas del Toro, Panama, in August 2012. The main objective of the study was to collect and describe algae species and know the vertical distribution formed in the roots. In order to know the algae vertical distribution, three zones were established in each root: the low zone going from the base of the root to $0.50 \mathrm{~m}$ in height, the average zone going from $0.50 \mathrm{~m}$ to $1.10 \mathrm{~m}$, and the high zone from 1.10 to $1.30 \mathrm{~m}$., based on Peña-Salamanca (2008). Eleven species of phylum Rhodophyta were identified, of which B. montagnei, B. moritziana, B. tenella, P. howei, P. scopulorum, A. spicifera, and C. caespitosa were the most outstanding. In addition, three species of phylum Chlorophyta were collected and identified: C. linum, B. verticillata, and Chlorodesmis sp. Regarding the vertical distribution of algae in the roots, most of the species were in the lower and middle areas of the

1 Escuela de Biología, Universidad Autónoma de Chiriquí. 0427. David, Chiriquí, Panamá.

2 Escuela de Biología, Facultad de Ciencias Naturales y Matemática, Universidad de El Salvador.

olga.tejada@ues.edu.sv**
\end{abstract}


root, while only $B$. montagnei was in the upper part. The study is expected to contribute to the phycoflora knowledge of Panama's Caribbean mangroves.

Keywords: Bostrychetium, roots, vertical distribution, tolerance to salinity, Caribbean Sea

\section{INTRODUCCIÓN}

El bosque de manglar constituye un hábitat propicio para la procreación, nutrición, refugio y permanencia de numerosas especies marinas y terrestres. El arreglo enmarañado de las raíces fúlcreas del mangle rojo $R h i$ zophora mangle Linneo constituyen un substrato firme y seguro para los organismos que en ellas habitan; en cuanto a las comunidades de algas e invertebrados epibiontes, las raíces brindan una amplia superficie húmeda disponible para la colonización; sin embargo, el manglar impone retos ambientales importantes a estos organismos, debido a las fluctuaciones de humedad, salinidad, luz, temperatura y concentración de nutrientes durante las mareas bajas (Planas et al. 2013). Para conservar el agua que usan en sus principales funciones vitales, algunas especies han modificado su aparato respiratorio y excretor; otras, sus patrones de comportamiento y distribución, (Lüning, 1990; Ruiz \& López-Portillo, 2014; 2017).

A través de estudios eco fisiológicos se ha comprobado que la marcada tolerancia de las algas de manglar a las variaciones de salinidad y períodos de desecación se debe a su capacidad de sintetizar, acumular y regular compuestos orgánicos que favorecen la competencia iónica, ya que estos actúan como solutos compatibles al interior de las células, lo que protege a las macromoléculas y su actividad enzimática ante los incrementos de salinidad, (Karsten et al. 1994 \& Planas et al. 2013). Las especies Bostrychia radicans (Montagne) y Caloglossa lepriurii (Montagne) Martens, por ejemplo, sintetizan polialcoholes que facilitan sus procesos de osmorregulación, (Mann \& Steinke, 1988; Tejada 2002; Peña-Salamanca, 2008).

Las comunidades de algas epibiontes de las raíces de $R$. mangle son la base de la cadena alimenticia de diversos organismos del medio acuático y también brindan un aporte importante de materia orgánica para la producción de detritos que se transportan hacia los bosques adyacentes, (Planas et al. 2013).

Se ha demostrado que estas comunidades también poseen un importante valor ecológico, por su aporte de carbono y materia orgánica disuelta en la columna de agua; así mismo, por la capacidad que muestran de remover nitrógeno, fósforo, metano y metales pesados que afectan la calidad de las aguas costeras, (Marín Salgado \& Peña Salamanca, 2016).

Diversos autores sostienen que la mayoría de los estudios de algas de manglar han sido realizados en las 
zonas subtropicales, y ha sido poca la atención dedicada a estas comunidades algales en las zonas tropicales, (Suárez, 1989; Averza et al. 2002; Barrios et al. 2003; Peña-Salamanca, 2008).

Sobre esto, Zucarello et al. (2012), refieren que en América Central son pocos los análisis relacionados con algas de manglar que se han realizado y destacan los de Pedroche et al. (1995) en el Pacífico de México y Guatemala y el de Tejada (2008) en los manglares de El Salvador. También, los autores mencionan que el resto de estas especies, asociadas a bosques de manglar, son informadas de manera general en el Checklist de las algas del Pacífico de Centroamérica (Fernández-García et al. 2011).

En el caso de Panamá, Rodríguez et al. (2010), realizaron un estudio exploratorio de las especies de macroalgas que crecen asociadas a la vegetación del manglar de Remedios, al sureste de la provincia de Chiriquí; Panamá. En este país, no existen antecedentes de otros estudios de este tipo, realizados en los manglares del Pacífico o del Caribe.

El objetivo principal del estudio que se presenta, fue recolectar y describir las especies de algas que crecen asociadas a los árboles de $R$. mangle en el manglar de playa Estrella, en el Caribe de Panamá y conocer la distribución vertical que las algas establecen en las raíces. Se espera que el estudio, contribuya a incrementar el conocimiento de la biodiversidad algal de Panamá.

\section{MATERIALES Y MÉTODOS}

\section{Descripción del área de estudio}

En agosto de 2012 se realizó un estudio descriptivo de las especies de macroalgas que crecen asociadas a las raíces de $R$. mangle en un pequeño parche de manglar ubicado en playa Estrella de la isla Colón, distrito de Bocas del Toro, provincia de Bocas del Toro, Panamá, entre los $09^{\circ} 24^{\prime} 19.026^{\prime \prime} \mathrm{N}$ y $82^{\circ}$ 19’ 28.176" W (Fig. 1).

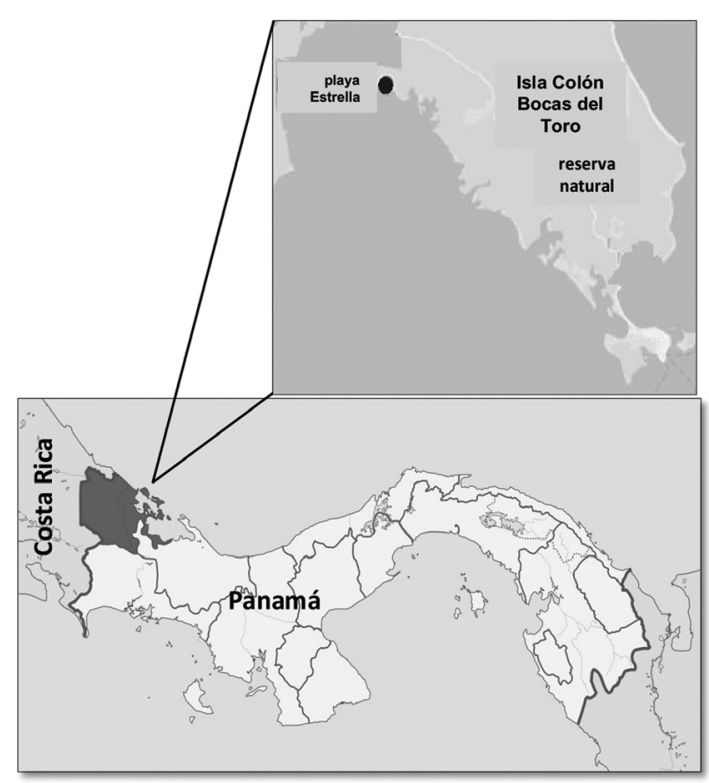

Fig. 1. Mapa de Panamá y ubicación de playa Estrella, isla Colón, distrito de Bocas del Toro, provincia de Bocas del Toro. (https://es.wikipedia.org y Google Map 2019)

Fig. 1. Map of Panama showing the location of Playa Estrella, Colón Island, Bocas del Toro district, Bocas del Toro province. (https:// es.wikipedia.org y Google Map 2019) 
La metodología implementada para recolectar los especímenes consistió en un recorrido de 6 horas para inspeccionar las raíces y cortezas de los árboles de R. mangle; así mismo, se aprovechó para determinar la distribución vertical que las algas establecen en el sitio; para lo que se definió, a lo largo de cada raíz, tres zonas: zona baja, que va desde la base de la raíz hasta $0.50 \mathrm{~m}$ de altura, zona media de $0.50 \mathrm{~m}$ a $1.10 \mathrm{~m}$ y zona alta, de 1.10 a $1.30 \mathrm{~m}$, este criterio se basó en la metodología empleada por Peña-Salamanca (2008).

Para la identificación taxonómica de las algas hasta el taxón más bajo posible, se estudiaron caracteres morfológicos distintivos, también se observaron cortes histológicos a la luz de un microscopio de campo claro marca Labomed modelo LX 400 y se utilizaron las claves taxonómicas de Taylor (1945 y 1960), Littler et al. (1989); Littler \& Littler (2000), Abbott (1999), publicaciones donde se describen especies asociadas a manglares. Además, se consultó la base de datos AlgaeBase (Guiry \& Guiry, 2019), para descripciones de especies, información taxonómica, y los datos de la distribución geográfica de especies.

\section{RESULTADOS}

Se identificaron y describieron un total de 14 especies (Cuadro 1), de las cuales, 11 pertenecen el phylum Rhodophyta incluidas en cinco familias, de ellas la más diversa fue la familia Rhodomelaceae con seis especies: Bostrychia montagnei Harvey (Fig. 2ef), B. moritziana (Sonder ex Kützing) J. Agardh (Fig. 6a y b), B. tenella (J. V. Lamouroux) J. Agardh (Fig. 3c y d), Polysiphonia howei Hollenberg (Fig. 4a, b, c y d), P. scopulorum Harvey (Fig. 2c y d) y Acanthophora spicifera (Vahl) Børgesen (Fig. 3a y b); la familia Ceramiaceae estuvo representada por Centroceras minutum Y. Yamada y Ceramium cimbricum H. E. Petersen (Fig. 5c y d); de la familia Cystocloniaceae, Hypnea cf spinella (C. Agardh) Kützing (Fig. 5b), Catenella caespitosa (Withering) L. M. Irvine, de la familia Caulacanthaceae (Fig. 3e y f ) y Heterosiphonia sp. Montagne de la familia Dasyaceae (Fig. 5d y e). Las especies del Phylum Chlorophyta encontradas fueron Chaetomorpha linum (O. F. Müller) Kützing (Fig. 2a) de la familia Cladophoraceae; Boodleopsis verticillata $\mathrm{E}$. Y. Dawson (Fig. 2b) y Chlorodesmis sp Harvey \& Bailey (Fig. 4e, f) ambas de la familia Udoteaceae. 
Cuadro 1. Especies de algas asociadas a raíces de árboles de $R$. mangle en playa Estrella, Bocas del Toro, Panamá

Table 1. Algae species associated with $R$. mangle tree roots in Estrella beach, Bocas del Toro, Panama

\begin{tabular}{|c|c|c|c|}
\hline Phylum & Orden & Familia & Especie \\
\hline \multirow[t]{11}{*}{ Rhodophyta } & Ceramiales & Rhodomelaceae & $\begin{array}{l}\text { Acanthophora spicifera (Valh) } \\
\text { Borgesen }\end{array}$ \\
\hline & Ceramiales & Rhodomelaceae & Bostrychia montagnei Harvey \\
\hline & Ceramiales & Dasyaceae & Heterosiphonia sp. Montagne \\
\hline & Ceramiales & Rhodomelaceae & $\begin{array}{l}\text { Bostrychia moritziana (Sonder } \\
\text { ex Kützing) J. Agardh }\end{array}$ \\
\hline & Ceramiales & Rhodomelaceae & $\begin{array}{l}\text { Bostrychia tenella (J.V. } \\
\text { Lamouroux) J. Agardh }\end{array}$ \\
\hline & Ceramiales & Rhodomelaceae & Polysiphonia howei Hollenberg \\
\hline & Ceramiales & Rhodomelaceae & $\begin{array}{l}\text { Polysiphonia scopulorum } \\
\text { Harvey }\end{array}$ \\
\hline & Gigartinales & Caulacanthaceae & $\begin{array}{l}\text { Catenella caespitosa } \\
\text { (Withering) L. M. Irvine }\end{array}$ \\
\hline & Ceramiales & Ceramiaceae & $\begin{array}{l}\text { Centroceras minutum Y. } \\
\text { Yamada }\end{array}$ \\
\hline & Ceramiales & Ceramiaceae & $\begin{array}{l}\text { Ceramium cimbricum H. E. } \\
\text { Petersen }\end{array}$ \\
\hline & Gygartinales & Cystocloniaceae & $\begin{array}{l}\text { Hypnea cf spinella (C. Agardh) } \\
\text { Kützing }\end{array}$ \\
\hline \multirow[t]{2}{*}{ Chlorophyta } & Bryopsidales & Udoteaceae & $\begin{array}{l}\text { Boodleopsis verticillata E.Y. } \\
\text { Dawson } \\
\text { Chlorodesmis sp. Harvey \& } \\
\text { Bailey }\end{array}$ \\
\hline & Cladophorales & Cladophoraceae & $\begin{array}{l}\text { Chaetomorpha linum } \\
\text { (O.F.Müller) Kützing }\end{array}$ \\
\hline
\end{tabular}




\section{$\because$ REVMAR}
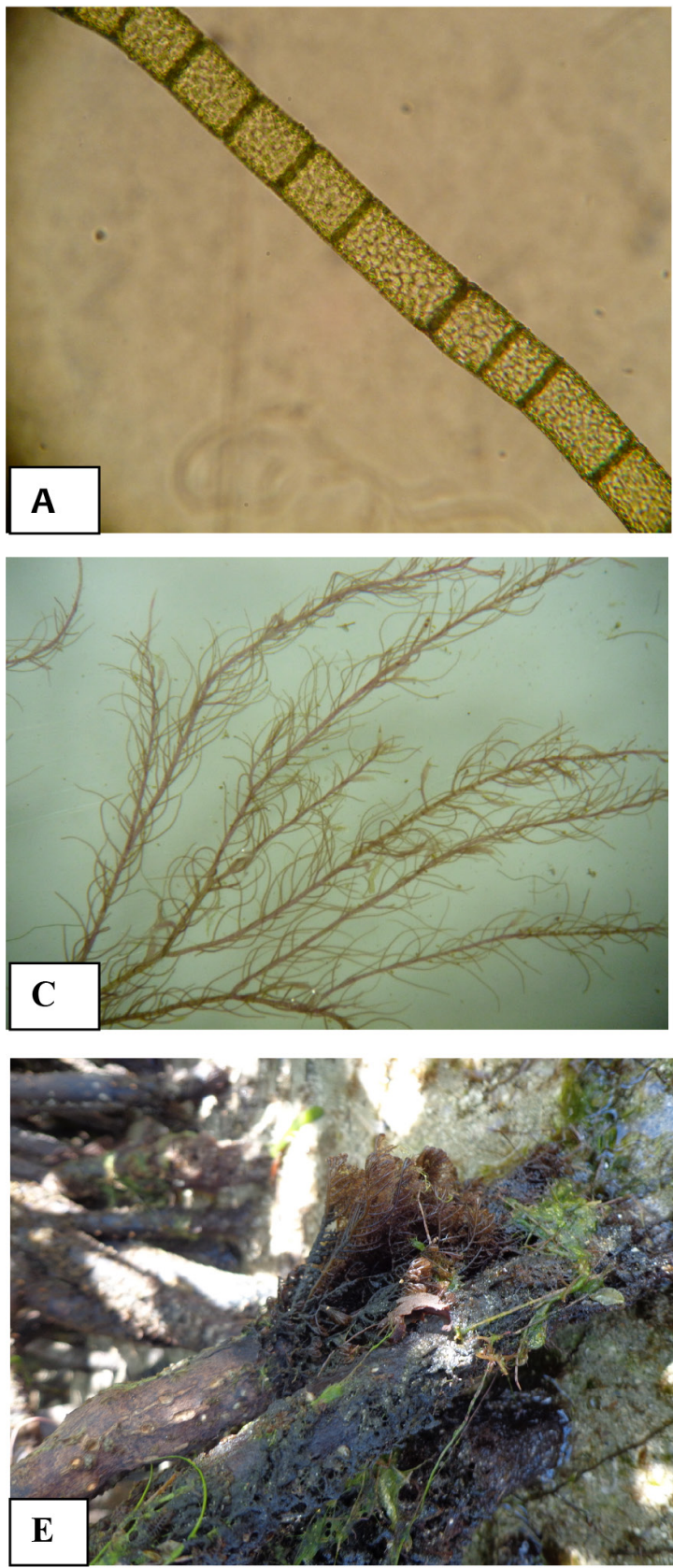

Rosemary Rios, Olga L. Tejada, Diana Morales, Elvia Miranda y Angélica Cerrud
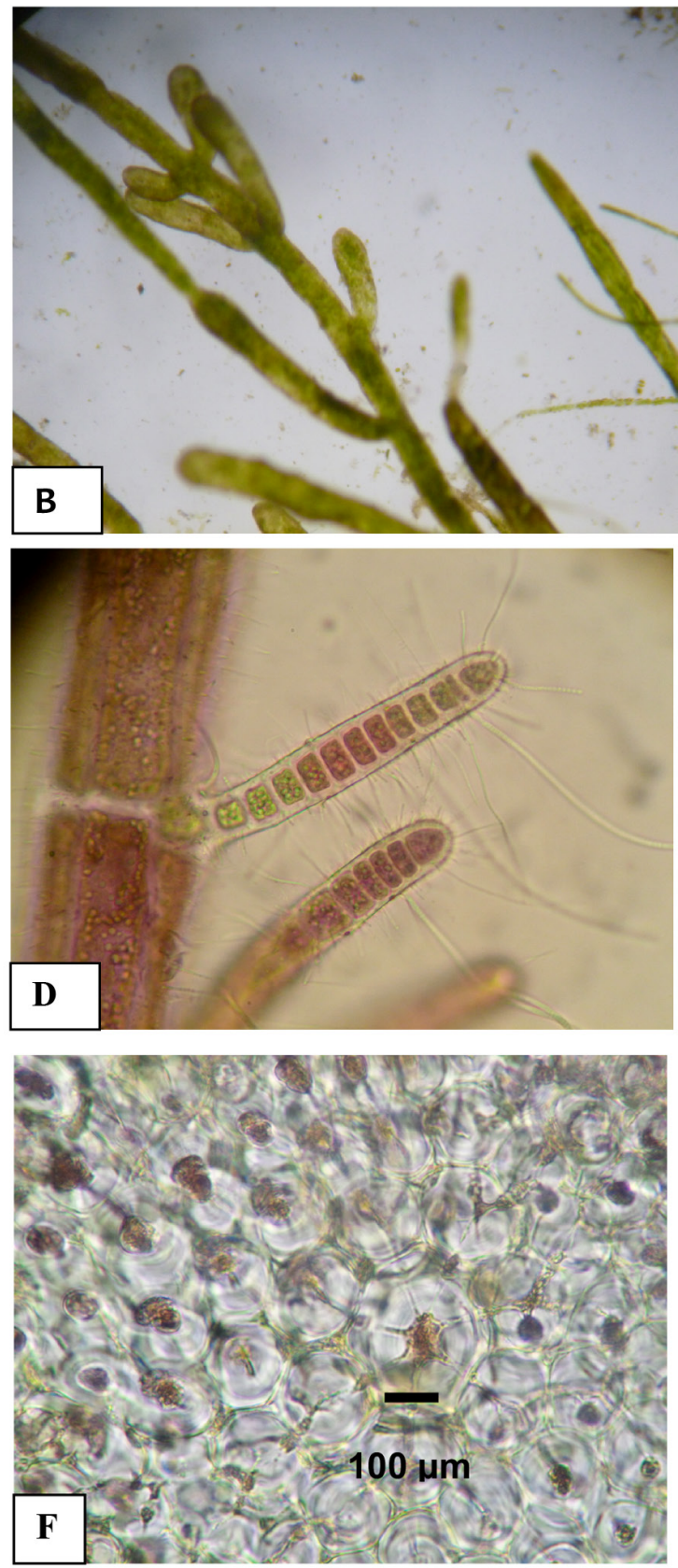
Comunidad de algas del manglar de playa Estrella, isla Colón, Bocas del Toro,

Panamá

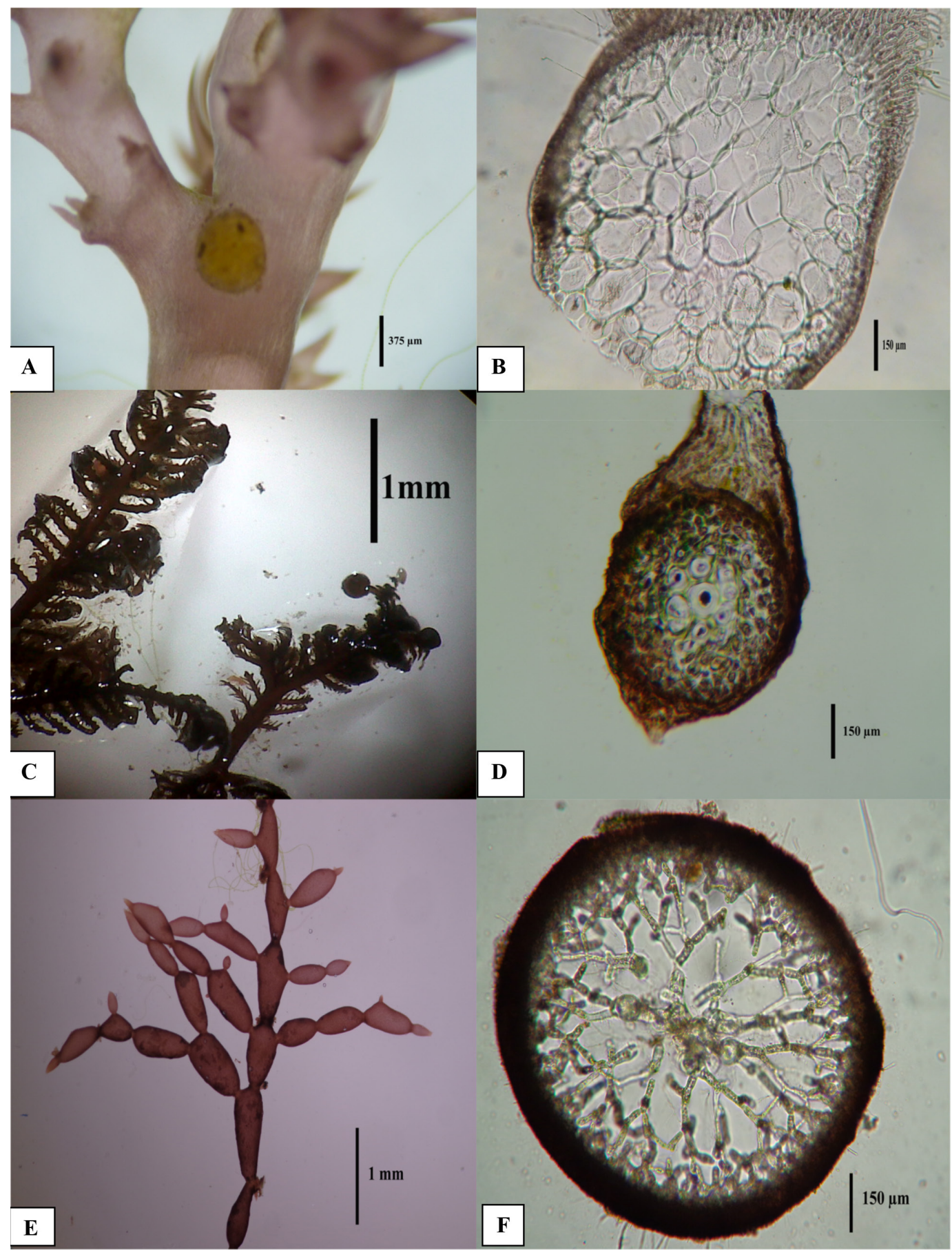

Rev. Mar. Cost. Vol. 11 (1): julio-diciembre 2019. 


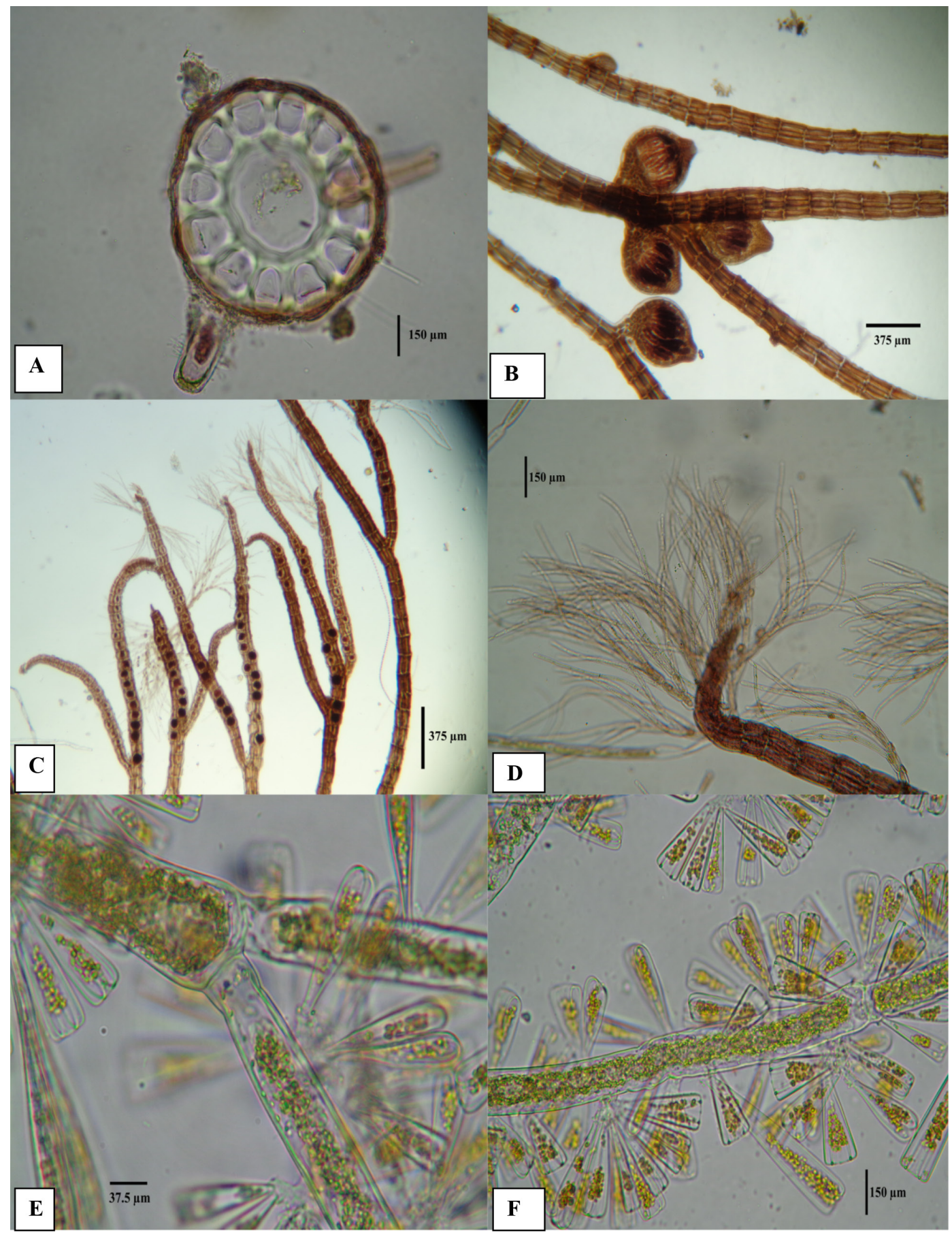


A Fig. 2. Chaetomorpha linum (A) Filamentos uniseriados septados cilíndricos con cloroplastos parietales reticulados $(10 \mathrm{x})$; Boodleopsis verticillata (B) Talos sifonales, fuertemente constrictos en la base de las ramificaciones verticiladas (10 x); Polysiphonia scopulorum (C) Talo filamentoso, ramificación alterna, talo sin corticación (D) Filamento erecto mostrando crecimiento apical y vellos absorbentes $(10 \mathrm{x})$; Bostrychia montagnei (E) sobre raíces de R. mangle; (F) Corte transversal mostrando células medulares y 6 células pericentrales alrededor de eje axial $(40 \mathrm{x})$
Fig. 2. Chaetomorpha linum (A) Cylindrical septate uniseriate filaments with reticulate parietal chloroplasts $(10 \mathrm{x})$; Boodleopsis verticillata (B) Siphonal thallus, strongly constricted in the base of the verticillate branches $(10 \mathrm{x})$; Polysiphonia scopulorum (C) Filamentous thallus, alternating branching, thallus without cortication (D) Erect filament showing apical growth and absorbent hairs $(10 \mathrm{x})$; Bostrychia montagnei(E) on R. mangle roots; (F) Cross section showing medullary cells and 6 pericentral cells around the axial axis $(40 \mathrm{x})$
Fig. 3. A-B: Acanthophora spicifera. (A) Cistocarpo (B) Corte transversal mostrando fuerte corticación. C-D: Bostrychia tenella. (C) Ramificación pinnada y cistocarpo (D) Corte transversal mostrando las células pericentrales.E-F:Catenellacaespitosa. (E) Talo y ramas pseudodicotómicas. (F) Corte transversal mostrando la médula en forma de collarines hacia el centro y las cinco células pericentrales alrededor del eje central
Fig. 3. A-B: Acanthophora spicifera. (A) Cystocarp (B) Cross section showing strong cortication. C-D: Bostrychia tenella. (C) Pinnate branch and cystocarp (D) Cross section showing pericentral cells. E-F: Catenella caespitosa. (E) Thallus and pseudo-dichotomous branches. Cross section showing the medulla in the form of collars toward the center and the five pericentral cells around the central axis.
Fig. 4. A-D: Polysiphonia howei (A) Corte transversal, ausencia de corteza. Cistocarpos. (B) Tetrasporangios (C) Filamentos apicales (D). E-F: Chlorodesmis sp. (E) Filamentos ramificados con cloroplastos reticulados, constricciones basales mostrando falsa dicotomía. (F) Diatomeas del género Licmophora sp., unidas a los filamentos.
Fig. 4. A-D: Polysiphonia howei (A) Cross section, absence of bark. Cystocarps (B) Tetrasporangia (C) Apicalfilaments(D).E-F: Chlorodesmis sp. (E) Branched filaments with crosslinked chloroplasts, basal constriction showing false dichotomy. (F) Diatoms Licmophora sp., attached to the filaments 
En cuanto a la distribución ver- en las zonas baja y media de la raíz y tical de las algas, se observó que la solamente el alga $B$. montagnei en la mayoría de las especies se repartían parte alta (Cuadro 2).

Cuadro 2. Distribución vertical de las especies de algas epífitas en las raíces de Rhizophora $m$. en playa Estrella

Table 2. Vertical distribution of epiphytic algae species in roots of Rhizophora $m$. at Playa Estrella

\begin{tabular}{lll}
\hline Zona alta & Zona media & Zona baja \\
$\mathbf{1 . 1 0} \mathbf{1 . 3 0} \mathbf{~ m}$ & $\mathbf{0 . 5 0} \mathbf{~} \mathbf{a} \mathbf{1 . 1 0} \mathbf{~ m}$ & Desde la base hasta $\mathbf{0 . 5 0} \mathbf{m}$ \\
\hline Bostrychia montagnei & Acanthophora spicifera & Polysiphonia scopulorum \\
& Heterosiphonia sp. & Polysiphonia howei \\
& Bostrychia moritziana & Catenella caespitosa \\
Bostrychia tenella & Centroceras minutum \\
& Polysiphonia howei & Ceramiun cimbrincum \\
& Catenella caespitosa & Hypnea spinella \\
& Boodleopsis verticillata & Chlorodesmis sp. \\
& & Chaetomorpha linum \\
\hline
\end{tabular}
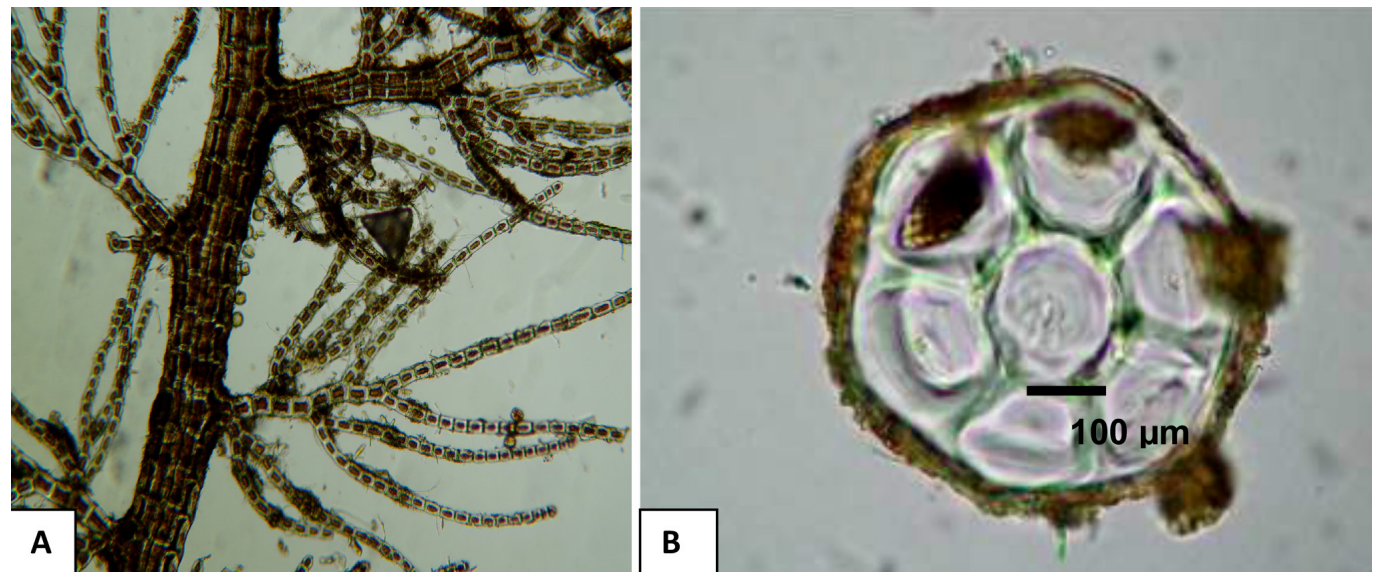

Fig. 6. Bostrychia moritziana. (A) Talo suave con ramificación regularmente alterna, patrón que se repite en las ramificaciones de segundo orden. (B) Corte transversal, células corticales ausentes, con seis células pericentrales alrededor del eje central

Fig. 6. Bostrychia moritziana (A) Soft thallus with regularly alternating ramification, pattern repeated in the ramifications of second order (B) Cross section, absent cortical cells, with six pericentral cells around the central axis 


\section{Descripción de las especies}

Bostrychia montagnei Harvey (Fig 2e-f).

Talos que forman densos cojinetes de color púrpura oscuro. Los ejes principales son erectos con ramificación alterna. Las ramas son polisifónicas en la base y parte media, pero hacia las puntas se alargan y se vuelven monosifónicas. Anatómicamente, los ejes principales están rodeados de 6 a 7 células pericentrales y de una fuerte corticación.

Distribución geográfica. Bermuda, Florida, Luisiana, México, Belice, El Salvador, Panamá, Bahamas, Cuba, Jamaica, Atlántico occidental, Brasil y Sierra Leona.

Bostrychia moritziana (Sonder ex Kützing) J. Agardh (Fig. 6a y b)

Talos finos que pueden alcanzar hasta $5 \mathrm{~mm}$ de largo, son suaves al tacto, de color rojo intenso, constituidos por un eje central formados de 7 a 8 células pericentrales, sin corticación. La ramificación es regularmente alterna, hasta de $2 \mathrm{~mm}$ de longitud, las ramitas se vuelven uniseriadas y se curvan hacia el ápice. Estas algas se adhieren al sustrato por medio de hápteros en la zona ventral del eje o estolón.

Distribución geográfica. Florida, Georgia, Carolina del Norte y Carolina del Sur, Texas; Guatemala, El Salvador, Costa Rica, islas del Caribe, Atlántico occidental, Colombia, Guyana, Venezuela, África, islas del océano Índico, India, Pakistán, Arabia Saudita, Japón, China, Indonesia, Malasia, Tailandia,
Australia, Nueva Zelanda, islas del Pacífico.

Bostrychia tenella (J. V. Lamouroux) J. Agardh (Fig. 3c y d).

Talos postrados, que forman cojinetes de color púrpura oscuro casi negro, doblemente pinnada, con ápices encorvados. Tallas de 2 a $5 \mathrm{~cm}$ de largo. Ejes fijos por medio de hápteros que nacen en la cara inferior de las ramificaciones. El talo presenta de 6 a 8 células pericentrales rodeadas de 1 a 4 líneas de células corticales que tienden a hacerse muy pequeñas hacia la superficie de la corteza. Esta especie presenta numerosas ramitas curvadas, que se vuelven monosifónicas hacia los ápices.

Distribución geográfica. Bermuda, Florida, Belice, El Salvador, Costa Rica, Panamá, islas del Caribe, Atlántico occidental, islas Galápagos, Venezuela, Brasil, África, isla Navidad, Bangladesh, India, Pakistán, Sri Lanka, China, Indonesia, Australia, Nueva Zelanda, islas del Pacífico.

Polysiphonia howei Hollenber (Fig. 4a, b, c y d)

Se trata de talos finos, muy pequeños, postrados de color rojo intenso a café; generalmente forman parches enmarañados sobre el sustrato. De los ápices de los ejes postrados se forman talos erectos de $1 \mathrm{~cm}$ de alto, con un diámetro de 100 a 150 micrómetros. Los talos sin ramificación o con pocas ramitas laterales. El talo está construido por 10 a 12 células pericentrales alrededor del eje, y forma segmentos, 
generalmente más anchos, que largos. Un rasgo distintivo de esta alga es que muestra un desarrollo simultáneo de ejes postrados y erectos.

Distribución geográfica. Bermuda, Florida, Carolina del Norte, México, Costa Rica, Panamá, islas del Caribe, Atlántico occidental, Colombia, Venezuela, Brasil, África, islas del océano Índico, Sri Lanka, China, Japón, Indonesia, Australia, Nueva Zelanda, islas del Pacífico.

Polysiphonia scopulorum Harvey (Fig.2c y d)

Se trata de un alga pequeña, color café intenso con ejes que se alternan entre postrados y erectos. En la mayoría de los casos, las ramitas son simples y arqueadas. Los ejes postrados pueden tener 100 micrómetros de diámetro y forman segmentos isodiamétricos, los talos erectos de 65 micrómetros de diámetro poseen diferentes largos en una misma rama. Anatómicamente los talos están constituidos de 4 células pericentrales sin corticación.

Distribución geográfica. Europa, islas del Atlántico, Alaska, Columbia Británica, California, Florida, Oregón, Washington, México, Belice, Costa Rica, Cuba, Atlántico occidental, Brasil, África, islas del océano Índico, sureste de Asia, China, Corea, Filipinas, Vietnam, Australia, Nueva Zelanda, islas del Pacífico.

Acanthophora spicifera (Vahl) Børgesen (Fig. 3a y b)

Talo con textura cartilaginosa, frágil, espinoso, con tallas de 10 a 25 $\mathrm{cm}$ de alto, color variable que va de rosado intenso a púrpura. La ramificación es irregular, radial, escasa. Las ramillas son cortas y poseen espinas en forma de espuela, espinas de $0.5 \mathrm{~mm}$ de largo. Anatómicamente, los talos presentan muchos sifones centrales rodeados por una fuerte corticación.

Distribución geográfica. Bermuda, Florida, México, Belice, Guatemala, El Salvador, Costa Rica, Panamá, islas del Caribe, Colombia, Venezuela, Brasil, Atlántico occidental, islas del océano Índico y África.

\section{Centroceras minutum Y. Yamada}

Alga filamentosa, diminuta, de poco más de $1 \mathrm{~cm}$ de altura, color rosa intenso a rojo; generalmente epifita con porciones postradas cortas y ejes erectos que se originan del eje postrado o que surgen unilateralmente. Usualmente no se ramifican o rara vez sucede en primer orden, los ápices son un tanto romos. Los internudos son mucho más largos hacia la base. La estructura del talo consta de 6 a 8 células periaxiales. Estas algas muestran espinas conspicuas en las jóvenes y en las viejas están ausentes o se ven oscuras; se pueden encontrar hasta 10 espinas por nudo. Estas algas se adhieren al sustrato por medio de rizoides uniseriados que terminan en forma de disco.

Distribución geográfica. Florida, Panamá, Atlántico occidental, islas del océano Índico, China, Australia, Nueva Zelanda, islas del Pacífico.

Ceramium cimbricum H. E. Petersen (Fig. 5c y d)

Rev. Mar. Cost. Vol. 11 (1): 9-29, julio-diciembre 2019. ISSN: $1659-455 \mathrm{X} \cdot \mathrm{e}-\mathrm{ISSN}$ : $1659-407 \mathrm{X}$ DOI: http://dx.doi.org/10.15359/revmar.11-2.1 
Comunidad de algas del manglar de playa Estrella, isla Colón, Bocas del Toro, Panamá

\section{$\because$ REVMAR}

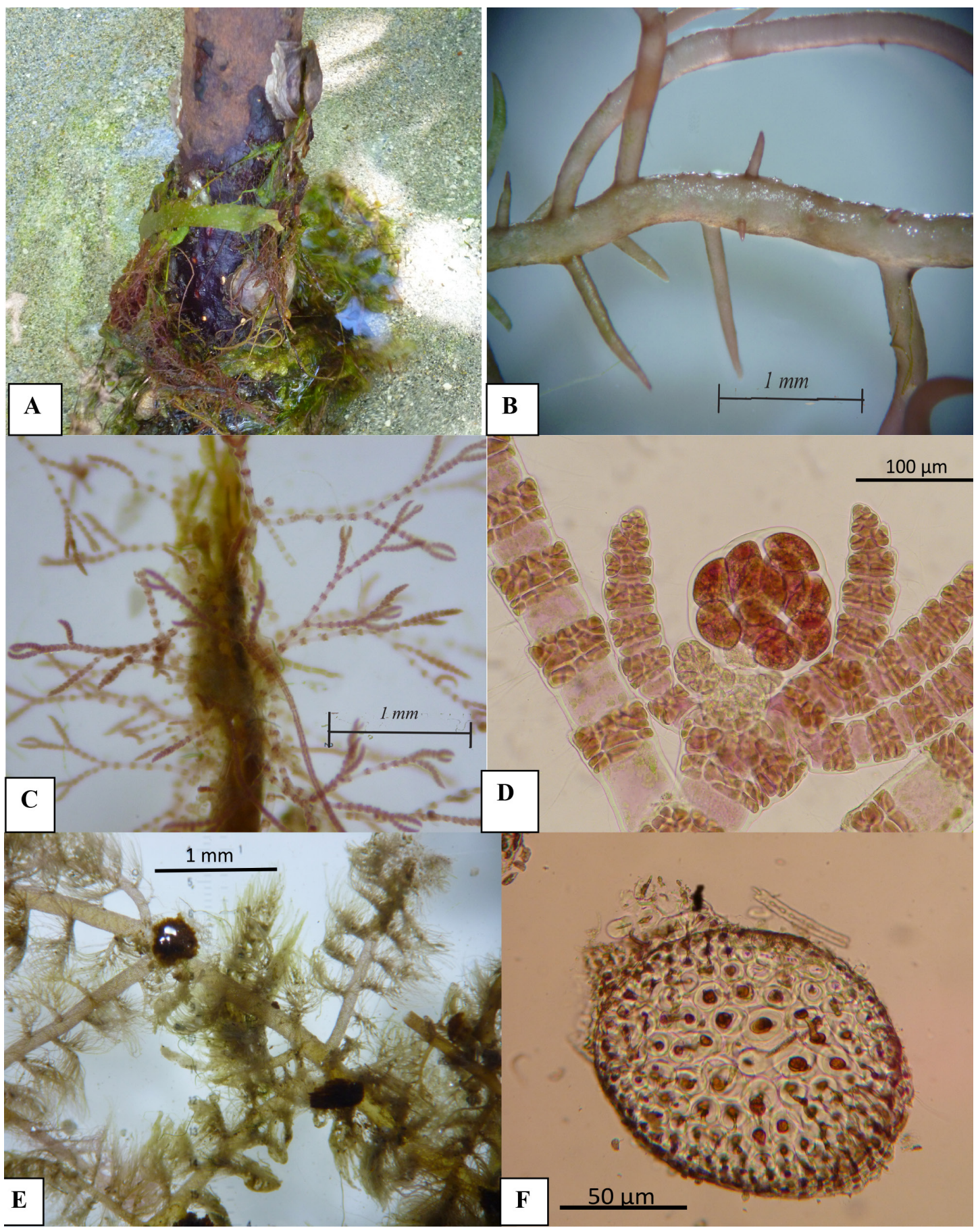

Rev. Mar. Cost. Vol. 11 (1): julio-diciembre 2019. 
4 Fig. 5. (A) Algas asociadas a raíz de Rhizophora mangle. (B). Hypnea $\mathrm{cf}$ spinella $(\mathrm{C})$ Talo de Ceramium cibricum (D) Tetrasporofito de C. cibricum (E) Talo de Heterosiphonia sp., teretes postrados ramificación principal alterna con ramitas subdicotomicas $(\mathrm{F})$. Corte transversal de la rama carente de corticación, son evidentes las células pericentrales alrededor del eje central (de seis a ocho)
Fig. 5. (A) Algae associated to Rhizophora mangle. (B) Hypnea of spinella (C) Thallus of Ceramium cibricum (D) Tetrasporophyte of $C$. cibricum(E)Thallus of Heterosiphonia sp. terete prostrate main branch alternates with subdichotomous twigs (F). Cross section of the branch lacking cortication; pericentral cells are evident around the central axis (from six to eight).
Talos de color rojo intenso, finos, delicados, producen penachos $\mathrm{y}$ alcanzan hasta $10 \mathrm{~cm}$ de alto, con ramificación irregularmente dicotómica, con ápices casi rectos y alargados, suele presentar ramificaciones laterales. Segmentos de 60 a 155 micrómetros de diámetro, hasta 600 micrómetros de largo. Las células de los nodos se observan en 2 bandas en los filamentos jóvenes y en los maduros de 4 a 6 bandas de células.

Distribución geográfica. Europa, islas del Atlántico, Alaska, Florida, Oregón, Texas, Cuba, Curazao, Atlántico occidental, Colombia, islas Galápagos, Venezuela, Sierra Leona, islas del océano Índico, sureste de Asia, China, Filipinas, Vietnam, Australia, Nueva Zelanda.

Hypnea of spinella (C. Agardh) Kützing (Fig. 5b)

El Talo crea matas enmarañadas de $3 \mathrm{~cm}$ de largo, coloración roja a café. Ramificación en distintos planos; las ramas son cilíndricas y terminan en forma de espina ligeramente curvadas; la ramificación es dicotómica abajo y alternas arriba. Anatómicamente el talo muestra células medulares de paredes delgadas, con formas irregulares u ovaladas densamente pigmentadas.

Distribución geográfica. Europa, islas del Atlántico, Florida, México, Belice, Costa Rica, islas del Caribe, Atlántico occidental, Colombia, islas Galápagos, Venezuela, Brasil, África, islas del océano Índico, sureste de Asia, China, Australia, Nueva Zelanda, islas del Pacífico.

Catenella caespitosa (Withering) L. M. Irvine (Fig. 3e y f)

Plantas muy pequeñas de color rosado intenso a morado; las ramas de 2 a 9 segmentos, dicotómicas o secundarias, a menudo se emiten desde la porción media del talo. Segmentos alargados ovales, aplanadas o cilíndricas, 
de 0.4-0.6 $\mathrm{mm}$ de diámetro, generalmente 3-5 veces más ancha que larga, estrecha entre los segmentos. Anatómicamente el talo está constituido de 5-6 células pericentrales, paredes con finos filamentos laterales en forma de collarines que se unen hacia el centro; también muestra de 2 a 4 capas de células corticales. Esta alga se adhiere al sustrato por medio de hápteros cortos, del lado ventral con constricciones que terminan en un amplio disco como almohadilla. El tetrasporangio es ovalado y se ubica en los segmentos terminales; los cistocarpos ovalados, 200-360 $\mu \mathrm{m}$ de diámetro se encuentran en los segmentos terminales.

Distribución geográfica. Europa, islas del Atlántico, Belice, Guatemala, El Salvador, Costa Rica, Panamá, Cuba, Trinidad y Tobago, Atlántico occidental, Colombia, islas Galápagos, Venezuela, África, Bangladesh Japón, Malasia, Filipinas, Nueva Caledonia. Heterosiphonia sp. Montagne (Fig. 5d y e)

El alga de color rosa intenso con ejes erectos que alternan con ejes postrados. La ramificación es dicotómica o sub dicotómica. El crecimiento del talo es simpodial y está constituido de 4 a 12 células pericentrales que rodean al eje central, los talos son teretes o comprimidos, y pueden presentar o no corticación.
Chaetomorpha linum (O. F. Müller) Kützing (Fig. 2a)

Alga filamentosa de color verde-amarillo, cetácea al tacto, que genera mechones. Los filamentos uniseriados un poco rizados de $1 \mathrm{~mm}$ de largo, cilíndricos tabicados y con cloroplastos parietales reticulados. Un carácter distintivo es que las células son más largas que anchas, con paredes delgadas. Sus dimensiones son 100-150 $\mu \mathrm{m}$ de ancho por 200-300 $\mu \mathrm{m}$ de largo.

Distribución geográfica. Europa, islas del Atlántico, Norte América, Costa Rica, Panamá, islas del caribe, Atlántico occidental, Colombia, islas Galápagos, Venezuela, Perú, Argentina, Brasil, Chile, África, islas del océano Índico, sureste de Asia, China, Australia, Nueva Zelanda, islas del Pacífico.

Boodleopsis verticillata E. Y. Dawson (Fig. 2b)

Talo filamentoso, sifonal con apariencia de alfombras sueltas o mechones entrelazados de color verde brillante sobre los sustratos. Los filamentos de 50 a $100 \mu \mathrm{m}$ diámetro están fuertemente constrictos en la base de las ramas. La ramificación puede ser dicotómica, tricotómica, pero prevalece la verticilada (espiral); sifones grandes, a menudo sin pigmentación; punta de crecimiento ligeramente bulbosa.

Distribución geográfica. Florida, México, El Salvador, Costa Rica, Panamá, islas del Caribe, Atlántico occidental, China, Filipinas. 
Chlorodesmis Harvey \& Bailey (Fig. $4 \mathrm{e}, \mathrm{f}$ )

Es un alga filamentosa sifonales de color verde brillante, forma cojinetes. Los talos pueden alcanzar hasta 17 $\mathrm{cm}$. de alto, muestran ramificación dicotómica, no septados, de 75-515 $\mu \mathrm{m}$ de diámetro, que contienen numerosos núcleos, y cloroplastos en arreglo reticular. Sifones distales constreñidos en uno o ambos sitios por encima de las dicotomías. Se unen al sustrato por medio de rizoides incoloros, de diámetro menor que el de los talos o sifones fotosintéticos no consolidados.

\section{DISCUSIÓN}

Según Lizano (2006), a diferencia del Pacífico, en el mar Caribe de Centroamérica el ámbito de mareas es bajo, lo que explica que los factores ambientales como humedad, salinidad, irradiación y temperatura son más estables, lo cual se traduce en un bajo estrés para la población $\mathrm{y}$, por lo tanto, en una mayor diversidad y abundancia de algas y epibiontes en general. Así mismo, existe mayor disponibilidad de sustratos duros, entre ellos corales, piedras calizas y raíces de mangle; las salinidades son estables y la transparencia del agua es mayor (Cordeiro-Marino et al. 1992). Similar condición es registrada por el estudio de Barrios et al. (2003) en el golfo de Santa Fe en el caribe de Venezuela y el de Pinheiro-Joventino y Lima-Verde (1988) en la región noroeste de Brasil. En contraste, las comunidades de algas de manglar en las aguas costeras del océano Pacífico, son menos diversas y pobres en especies, por estar sometidas a mayor estrés ambiental, derivado del amplio ámbito de mareas, que las deja a la intemperie por horas, aumenta la salinidad y la desecación de sus talos (Cedeño 2009). Marín Salgado y Peña Salamanca (2016), mencionan que existe consenso entre diversos autores, quienes aseguran que la baja diversidad de comunidades de algas en las costas del Pacífico está vinculada a un clima desfavorable y talos relativamente poco desarrollados.

La diversidad de especies encontradas en el manglar de playa Estrella, demuestra que aquellas especies que generalmente habitan en espacios protegidos en aguas calmas y poco profundas de playas rocosas y arrecifes de coral, como es el caso de A. spicifera, Heterosiphonia sp, $C$. minutum, C. cimbricum, $H$. spinella, Cladophoropsis sp y $C$. linum, se han logrado establecer con éxito en las raíces de mangle rojo, situación que es coincidente con lo registrado por Littler \& Littler (2000) y por Marín Salgado y Peña Salamanca (2016) para los manglares del Caribe.

En el caso de las algas rojas de los géneros Bostrychia, Catenella y Polysiphonia, su amplia distribución 
en los manglares del Caribe y del Pacífico podría obedecer a que se trata de especies eurihalinas que se desarrollan bien en aguas hipohalinas e hipersalinas que inclusive exceden a la concentración de sal del agua de mar (Barrios et al. 2003).

La presencia Bostrychia y $\mathrm{Ca}$ tenella asociadas con otras algas rojas coinciden con las encontradas por Inclán (1989) en Quintana Roo, en el caribe de México y el de Suárez (1989) en Cuba, en donde se señala que las algas rodófitas fueron dominantes en las raíces de $R$. mangle. Por su parte, Peña-Salamanca (2008), afirma que la comunidad de algas epífitas de manglar en el Pacífico está dominada, principalmente, por algas rojas asociadas conocida como complejo Bostrychietum, que se refiere principalmente a la relación característica de cuatro géneros: Bostrychia, Catenella, Stictosifonia y Caloglossa. Así mismo Tejada (2002), quien estudió las algas de cinco manglares a lo largo de un gradiente climático en el pacífico de Costa Rica, afirma que diferentes especies de los géneros Bostrychia, Catenella y Caloglossa, constituyen la flora que domina en las raíces de $R$. mangle y que estas mismas asociaciones algales, son sobresalientes en los manglares de El Salvador donde las fluctuaciones de los paramentos ambientales son muy marcadas (Tejada, 2013).
En cuanto a la distribución de las algas a lo largo de las raíces, en el manglar de playa Estrella, el mayor número de especies fueron encontradas en las zonas baja y media de la raíz. $B$. montagnei fue la única encontrada en la parte alta de la raíz.

De acuerdo con Peña-Salamanca (2008), la distribución del Bostrychietum en el ecosistema de manglar se restringe al estrato inferior de las raíces de $R$. mangle, donde se ve directamente afectado por factores ambientales como: las fluctuaciones de salinidad e intensidad luminosa, incrementos de temperatura, desecación, inundación de mareas y frecuencia de lluvias. En el manglar de playa Estrella la mayoría de las especies de algas encontradas en las raíces de $R$. mangle resultaron ser rojas principalmente en las secciones y media del sustrato. La preferencia de la distribución de macroalgas hacia la zona media de raíces de $R$. mangle, concuerda con la observación de Barrios et al. (2003), quienes sugieren como factores influyentes, la incidencia de rayos solares durante la bajamar, periodo en el que las algas son vulnerables. Géneros como Bostrychia presentan adaptaciones a las condiciones de estrés antes mencionadas, debido a que mantienen las tasas respiratorias y fotosintéticas bajas, que le confieren una mayor estabilidad (Mann \& Steinke 1988). Lo anterior, podría explicar que las especies 
de este género sean abundantes en los manglares de las costas del Pacífico, por ejemplo, Tejada (2002) informó las especies Bostrychia calliptera, $B$. pinnata, B. moritziana, B. pilulifera y $B$ radicans para los manglares del pacífico de Costa Rica; para El Salvador, Tejada (2013) informa las especies $B$. calliptera, B. moritziana, B. tenella y $B$ radicans y Peña-Salamanca (2017) informa las especies $B$. binderi, $B$. calliptera, B. moritziana, B. pinnata, $B$. radicans y $B$. tenella para las costas del pacífico de Colombia. En el manglar de playa Estrella, Bostrychia estuvo representada solamente por tres especies: $B$. tenella, $B$. moritziana y $B$. montagnei, esta última, ya había sido informada para el pacífico de Panamá, en el manglar de Remedios por Rodríguez et al. (2010).

La comunidad de algas asociadas a las raíces sumergidas de $R$. mangle juegan un papel importante dentro del ecosistema, debido a la gran variedad de epibiontes que se alimentan de ellas o que simplemente encuentran un microhábitat para reproducirse y protegerse de sus depredadores. Al remover la cobertura algal, es frecuente encontrar pequeños moluscos, crustáceos, isópodos, anélidos, etc., que a su vez sirven de alimento a peces, cangrejos y langostas favorecen el intercambio de materia y energía dentro del ecosistema (Márquez et al. 2006; Planas et al. 2013). En el man- glar de playa Estrella, las especies $B$. montagnei, A. spicifera, $P$. scopulorum, $B$. verticillata y $B$. tenella, presentaron poblaciones de crustáceos y gasterópodos. Algunos investigadores sostienen que esta fauna es de gran importancia en las redes tróficas y que sustenta los niveles tróficos más altos. Planas et al. (2013) comprobaron que las raíces de mangle rojo y las algas asociadas brindan un sustrato adecuado para el desarrollo y reproducción de diversas especies de macroinvertebrados, lo que permite concluir que la protección de los lechos de algas, asociados a las raíces es importante para disminuir la pérdida de recursos hidrobiológicos que sostienen la pesca artesanal.

Las descripciones hechas de las especies recolectadas, constituye una línea base de utilidad a futuras investigaciones relacionadas a la sistemática de la ficoflora de Panamá, ya que aún existe una falta de consenso taxonómico de algunas especies tanto para las costas del Pacífico como para las del Caribe. Así mismo, se puede profundizar en el estudio de las interrelaciones que las algas establecen con otros epibiontes y el servicio ecológico que éstas brindan en los manglares de Panamá.

\section{AGRADECIMIENTOS}

Expresamos nuestro agradecimiento a Orlando Cáceres, coordinador de la Maestría en Biología Vegetal de la 
Escuela de Biología de la Universidad de Chiriquí, quién hizo posible el viaje a la isla Colón de Bocas del Toro y el valioso apoyo logístico de Rafael Rincón e Idalmi de Rincón, investigadores y curadores del herbario de la Universidad Autónoma de Chiriquí, Panamá y a los evaluadores del artículo, quienes con sus comentarios y aportes ayudaron a mejorar la calidad del mismo.

\section{BIBLIOGRAFÍA}

Abbott, I. (1999). Marine Red Algae of the Hawaiian Islands. Honolulu, H. EE. UU.: Bishop Museum Press.

Averza, A., Almodóvar, L. \& Martínez, A. (2002). Compendio bibliográfico de las algas del Caribe de Panamá: las algas verdes. Tecnociencia, 4, 141-160.

Barrios, J. E., Márquez, B. \& Jiménez, M. (2003). Macroalgas asociadas a Rhizophora mangle L. en el golfo de Santa Fe, Estado Sucre, Venezuela. Bol. Ins. Oceanogr. Venezuela., 42, 37-45.

Cedeño, J. (2009). Epibiontes asociados a las raices sumergidas del mangle rojo (Rhizophora mangle) de la laguna de Bocaripo, Guayacán, Estado Sucre, Venezuela. Tesis de grado no publicada, Universidad de Oriente, Núcleo de Sucre, Venezuela.

Cordeiro-Marino, M., Braga, M. R. A., Eston, V. R., Fujii, M. T. \& Yokoya, (1992). 3 - Mangrove Macroalgal Communities of Latin America: The State of Art and Perspectives, Editor(s): Ulrich Seeliger, In Physiological Ecology, Coastal Plant Communities of Latin America, Academic Press, (pp 51-64). New York, EE. UU.: Academic Press.
https://doi.org/10.1016/B978-0-08092567-7.50009-X

Fernández-García, C., Riosmena-Rodríguez, R., Wysor, B., Tejada, O. L. \& Cortés, J. (2011). Checklist of the Pacific marine macroalgae of Central America. Bot. Mar., 54, 53-73. https://doi. org/10.1515/bot.2011.001

Guiry, M. D., Guiry, M. D. \& Guiry, G. M. (2019). AlgaeBase. World-wide electronic publication, National University of Ireland, Galway. Recuperado en mayo 08, 2019, disponible en http:// www.algaebase.org.

Inclán, R. (1989). Ecología de la epibiosis en las raíces inmersas de Rhizophora mangle en Bahía de la Ascensión, Quintana Roo, México. Cien. Mar., 15(1), 1-20. https://doi.org/10.7773/cm.v15i1.630

Karsten. U., West, J. A., Zucarello, G. \& Kirst, G. O. (1994). Physiological ecotypes in the marine alga Bostrychia radicans (Ceramiales, Rhodophyta) from the east Coast of the U.S.A. J. Phycol., 30, 174-182. https://doi.org/10.1111/ j.0022-3646.1994.00174.x

Littler, D. S. \& Littler, M. M. (2000). Caribbean Reef Plants. Washington D. C. EE. UU.: Offshore Graphics Inc.

Littler, D. S., Littler, M. M., Bucher, K. E. \& Norris, J. N. (1989). Marine Plants of the Caribbean. A Field guide from Florida to Brazil. Washington. D. C. EE. UU.: Smithsonian Institution Press.

Lizano, O. (2006). Algunas características de las mareas en la costa Pacífica y Caribe de Centroamérica. Rev. Cienc. Tec., 24(1), 51-64.

Lüning, K. (1990). Seaweeds. their environment, biogeography, and ecophysiology. New York, EE. UU.: Jonh Wiley \& Sons, Inc. 
Mann, F. D. \& Steinke, T. D. (1988). Photosynthetic and respiratory responses of the mangrove associated red alga, Bostrychia radicans and Caloglossa lepriurii. S. Afr. J. Bot., 54(3), 203-

207. https://doi.org/10.1016/S02546299(16)31320-5

Marín Salgado, H. \& Peña Salamanca, E. J., (2016). Macroalgas bénticas de la Bahía de Tumaco, Pacífico colombiano. Hidrobiológica, 26(2), 299-309. https://doi.org/10.24275/uam/izt/dcbs/ hidro/2016v26n2/Marin

Márquez, B., Jiménez, M. \& Allen, T. (2006). Crustáceos asociados a las raíces del mangle rojo Rhizophora mangle (L.) en el golfo de Santa Fe, Estado Sucre, Venezuela. Ciencia, 14(1), 12-27.

Pedroche, F. F., West, J. A., Zuccarello, G. C., Senties, A. \& Karsten, U. (1995). Marine red algae of the mangroves in south Pacific México and Pacific Guatemala. J. Bot. Mar., 38, 111-119. https://doi. org/10.1515/botm.1995.38.1-6.111

Peña-Salamanca, E. (2008). Dinámica espacial y temporal de la biomasa algal asociada a las raíces de mangle en la bahía de Buenaventura, costa Pacífica de Colombia. Bol. Invest. Mar. Cost., 37(2), 55-70. https://doi.org/10.25268/ bimc.invemar.2008.37.2.191

Peña-Salamanca, E. (2017). El complejo Bostrychietum: la flora de algas asociadas a las raíces del manglar en la costa pacífica colombiana. Rev. Acad. Colomb. Cienc. Ex. Fis. Nat., 41(160), 338-348. https://doi.org/10.18257/raccefyn.485

Pinheiro-Joventino, F. \& Lima-Verde, N. G. (1988). Ocorrência e distribucão de macroalgas no Estuário do Rio Cocó, Fortaleza, Brasil. Arq. Ciên. Mar., 27, 83-89.
Planas, A., Tejada, O., Tejada, O. L., \& Guerrero, C. (2013). Los epibiontes en las raíces de Rhizophora spp. en el manglar Barra de Santiago, Departamento de Ahuachapán, El Salvador. Rev. Puente Biológico, 5, 11-49.

Rodríguez, E. Caballero, E., Gómez, A. Gómez, D. Rodríguez, R., \& Tejada, O. L. (2010). Algas del manglar de Remedios, Chiriquí, Panamá. Rev. Puente Biológico., 3, 71-87.

Ruiz, M. \& López-Portillo, J. (2014). Variación espacio-temporal de la comunidad de macro invertebrados epibiontes en las raíces del mangle rojo Rhizophora mangle (Rhizophoraceae) en la laguna costera de La Mancha, Veracruz, México. Rev. Biol. Trop., 62(4), 13091330. https://doi.org/10.15517/rbt. v62i4.12582

Ruiz, M. \& López-Portillo, J. (2017). Colonización y supervivencia de epibiontes sésiles en substratos artificiales similares a rizóforos de Rhizophora mangle (Rhizophoraceae) en La Mancha, México. Rev. Biol. Trop., 65(2), 745761. https://doi.org/10.15517/rbt. v65i2.25362

Suárez, A. M. (1989). Algas asociadas a las raíces de Rhizophora mangle L. en cayos al este de la Isla de la Juventud, Cuba. Rev. Inv. Mar., 10(2), 117-123.

Taylor, W. R. (1945). Pacific Marine Algae of the Allan Hancock Expedition to the Galapagos Islands. Los Angeles, EE. UU.: University of Southern California Press,

Taylor, W. R. (1960). Marine Algae of the Eastern Tropical and Subtropical Coasts of the Americas. Michigan, EE. UU.: University of Michigan Press. 
Tejada, O. L. (2002). Macroalgas asociadas a raices de mangle: costa pacifica de Costa Rica. Tesis de Maestría no publicada. Universidad de Costa Rica, San José, Costa Rica.

Tejada, O. L. (2008). Notas preliminares de la flora algal asociada a los bosques de manglar de El Salvador. Rev. Mesoam., 12, 1-193.

Tejada, O. L. (2013). Notas breves sobre macroalgas de los manglares de El Salvador. Rev. Bioma., 10(1), 6-12.
Zucarello, G. C., Kamiya, M., Ootsuki, R., Loiseaux de Goër, S., Pedroche, F. F. \& West, J. A. (2012). New records of red algae from mangroves in El Salvador and Pacific Mexico, combining culture and molecular observations. Bot. Mar., 55(1), 101-111. https://doi. org/10.1515/bot-2011-0075 
